\title{
Analysis of a single soybean microtubule's persistence length
}

\author{
Mitra Shojania Feizabadi*, Jimmy Barrientos, Carly Winton
}

Department of Physics, Seton Hall University, South Orange, USA

Email: ${ }^{*}$ shojanmi@shu.edu

Received 30 June 2013; revised 20 July 2013; accepted 15 August 2013

Copyright (C) 2013 Mitra Shojania Feizabadi et al. This is an open access article distributed under the Creative Commons Attribution License, which permits unrestricted use, distribution, and reproduction in any medium, provided the original work is properly cited.

\begin{abstract}
In this study, we have reported for the first time the persistence length of a single soybean microtubule in vitro. The measurement is based on analysis of thermal bending of a single soybean microtubule. Similar to mammalian microtubules, the length dependency of the persistence length was also observed for this species of plant microtubule. The identification of two intrinsic aspects of plant microtubules: the persistence length and the compositional structure in terms of variety of beta tubulin isotypes, and the potential correlation between these two factors, provides evidence for the functionality mechanism of plant microtubules. In this work, these two fundamental factors have been further discussed for our case study: single soybean microtubule.
\end{abstract}

Keywords: Microtubule; Soybean; Persistence Length; $\beta$ Tubulin Isotype

\section{INTRODUCTION}

Microtubules are key components of the cytoskeleton. In connection with other intracellular components, they are involved in many functions ranging from cell division, cell shape, chromosome movement during mitosis, and intracellular transport $[1,2]$. These hollow shaped polymers are dynamic and their unique mechanical and dynamic properties generate structure and forces that are essential to support the cellular functions [3,4]. The evidence indicates that mammalian and plant microtubules are distinguished from one another in terms of their involvements in intracellular functionality. For example unlike mammalian microtubules, plant microtubules are less engaged in intracellular transportation, while having a major role in cell expansion and cell shape. Further, microtubules are the dominant component of the plasma

\footnotetext{
Corresponding author.
}

membrane in plant cells while actin filaments play a similar role in mammalian cells, which indicates the key role of microtubules in plants' cell [5-8].

Microtubules are composed of $\alpha$ and $\beta$ tubulins. These bio filaments are intrinsically dynamic polymers that express a non-equilibrium behavior called dynamic instability, where they randomly switch between a growing and a shrinking phase, both in vitro and in vivo. This dynamic is recognized by the following parameters: growing and shrinking rates, which represent assembly and disassembly rates during the phases of growth and shrinkage, and the frequency of catastrophe and frequency of rescue, which represent the recurrence of switching from growing to shrinking and vice versa, respectively. Microtubules' dynamic instability, which has been the subject of many studies, has been confirmed both in mammalian and plant microtubules [4,9-13].

While this dynamic is common, the results of study by Moore et al. have shown that parameters of dynamic instability are different between plant and neural mammalian microtubules [7]. In that study, supported by the results of a study conducted by Panda et al. that indicates that microtubule dynamics are regulated by different isotype composition, different dynamic characterization of plant microtubules has been also associated with different isoforms from which microtubule is structured [7, 14]. Later, the correlation between dynamic instability and compositional structure was again confirmed by the reported result of a study conducted by Newton et al. [15]. This study focused on measuring dynamic parameters of specific non-neural mammalian microtubules polymerized from HeLa Cell tubulin in vitro, and reported different dynamic parameters and a slower dynamic instability as compared with Bovine brain microtubules. In addition, they reported that, unlike Bovine brain, HeLa Cell microtubules consist of a significantly different $\beta$ tubulin isotype distribution, and concluded that the slower dynamic instability can be a result of the diverse tubulin distribution. 
Different dynamic parameters of plant and mammalian microtubules are not the only factor which distinguishes them from one another. It is also notable that plant and mammalian microtubules show different sensitivity while interacting with anti-mitotic drugs. Different pharmacological properties across different mammalian microtubules and plant microtubules have also been associated with their diverse protein and compositional structure [16]. For example, the resistance to docetaxel in some of the breast cancer (MCF7) cells has been associated with the alteration of $\beta$ tubulin isotypes in the structure of MCF7 microtubules [17-20].

Many multi-dimensional research studies have been conducted to further clarify the differences between mammalian and plant microtubules. In these studies, however, more weight has been given to researches focused on dynamics of microtubules. In light of evidence provided by the outcomes of these studies the major differences between mammalian and plant microtubules are revealed. However, a number of questions are still open for further investigation concerning the better understanding of mechanical properties of microtubules in mammalian and non-mammalian microtubules.

Rigidity of microtubules is one of the mechanical properties dominant in functionality of a cell. The rigidity of neural microtubules has been measured by different methods [21-30]. The recent result of our study, on rigidity of a single microtubule polymerized from MCF7 tubulin with different beta tubulin structure, indicated a slight difference in rigidity of a single Bovine brain microtubule as a sample of neural microtubule, and MCF7 microtubule as a sample of non-neural microtubule, with different structural composition [31].

To further advance our knowledge about plant microtubules, the focus of this study is to measure the persistence length of a single soybean microtubule in vitro as species of a plant microtubule with different tubulin diversity in its structure, as compared to mammalian microtubules. Similar to our previous study, we chose to measure the rigidity of a single soybean microtubule based on monitoring the fluctuation of an individual microtubule in response to temperature, implementing the image analysis techniques.

\section{MATERIALS AND METHOD}

\subsection{Sample Preparation}

Microtubules were polymerized from soybean tubulin (Cytoskeleton, Denver, CO, Cat. TP005). As indicated, the tubulin is $90 \%$ purified in this kit. The tubulin at 5 $\mathrm{mg} / \mathrm{ml}$ was polymerized via incubation in BRB80 (80 mM PIPES, pH 7.0, 0.5 mM EGTA, and $1 \mathrm{mM} \mathrm{MgCl}$, and $1.0 \mathrm{mM}$ GTP with $10 \%$ Glycerol) at $37^{\circ} \mathrm{C}$. We observed the nucleation development and polymerization of soybean tubulin in a real-time sequence of 30 minutes.

To make an appropriate contrast, we also polymerized Bovine brain tubulin at $5 \mathrm{mg} / \mathrm{ml}$ (Cytoskeleton, Denver, $\mathrm{CO}, \mathrm{Cat}$. TL238). The tubulin purification in this kit is $99 \%$, with $10 \%$ Glycerol at the $37^{\circ} \mathrm{C}$, and observed on a regular basis over time.

$10 \mathrm{mg} / \mathrm{ml} \mathrm{BSA}$ was used to inhibit the microtubules from adhering to glass surfaces. To make a sample of polymerized microtubule after incubation, a few microliters, usually between $5 \mu \mathrm{l}$ to $7 \mu \mathrm{l}$, of this solution were pipetted onto a microscope slide $(75 \times 25 \times 1 \mathrm{~mm})$, with a $22 \times 22 \mathrm{~mm}$ cover slip (thickness No.1) placed on top. Pressure was applied to the top of the coverslip to reduce the thickness. The result was a solution depth of less than 4 micrometers. This state constrained the microtubules to move only in two dimensions. The edges of the coverslip were sealed by hot candle wax to avoid any fluid evaporation. All the experiments were conducted at room temperature $\left(24^{\circ} \mathrm{C}-25^{\circ} \mathrm{C}\right)$.

\subsection{Video Enhanced Differential Interference Contrast Microscopy and Image Analysis}

We visualized the samples using a differential interference contrast (DIC) microscope (Diphot 200, Nikon, Tokyo, Japan) equipped with a $100 \mathrm{X}$ oil-immersion objective lens $(\mathrm{NA}=1.25)$. The images and videos were recorded with a Sentech USB 2.0 camera (USA). The digitalized images were then taken at a rate of 1 frame every $10 \mathrm{~s}$.

The images were then analyzed by Image J (Rasband, W. S., Image J, US National Institutes of Health, Bethesda, Maryland, USA, http://rsb.info.nih.gov/ij/, 19972009). This software was used to adjust the enhance contrast between an individual microtubule and the background. It was used also to measure and monitor the coordinates of points along an individual microtubule in real time as part of the steps required for measuring the persistence length. The visualized soybean microtubules had lengths ranging from 15 to $21 \mu \mathrm{m}$.

\subsection{Mode Analysis and Bending Rigidity}

As expressed in the introduction, the rigidity of a single microtubule has been measured and reported through different methods. In our study we implemented a method introduced by Gittes et al. [21]. In this approach, the bending rigidity of a single microtubule is calculated by analyzing the shape fluctuation using the Fourier decomposition method. The method includes the estimation of errors correlated to the measurements. Following this method and to measure the length of a single microtubule, we first need to specify $M+1$ points along each individual microtubule. The pixel coordinates for each point are $\left(x_{m}, y_{m}\right)$. The length of each segment is determined 
using the following equation:

$$
\Delta S_{m}=\left[\left(x_{m+1}-x_{m}\right)^{2}+\left(y_{m+1}-y_{m}\right)^{2}\right]^{1 / 2}
$$

The tangent angle at each point will be found using the equation

$$
\theta\left(S_{m}\right)=\tan ^{-1}\left[\left(y_{m+1}-y_{m}\right) /\left(x_{m+1}-x_{m}\right)\right]
$$

The length of a microtubule can be calculated by:

$$
L=\sum_{m=1}^{M} \Delta S_{m}
$$

Since the fluctuations occur freely, the tangent angle can be decomposed to a large number of cosine Fourier modes:

$$
\theta(s)=\sum_{n=0}^{\infty} \theta_{n}(s)=\sqrt{\frac{2}{L}} \sum_{n=0}^{\infty} a_{n} \cos \left(\frac{n \pi}{L} S\right)
$$

where $a_{n}$ are the amplitudes. A single microtubule has an intrinsic curvature:

$$
\theta_{0}(s)=\sqrt{\frac{2}{L}} \sum_{n=0}^{\infty} a_{n}^{0} \cos \left(\frac{n \pi}{L} S\right)
$$

where $a_{n}^{0}$ is the mode amplitude corresponding to the filament intrinsic curvature. Therefore the mean amplitude is not zero.

The bending energy of a curved microtubule can be calculated by:

$$
\begin{aligned}
& U=\frac{1}{2} k \int_{0}^{L}\left(\frac{\partial \theta}{\partial S}-\frac{\partial \theta_{0}}{\partial S}\right)^{2} \mathrm{~d} s \\
& U=\frac{1}{2} k \sum_{n=1}^{\infty}\left(\frac{n \pi}{L}\right)^{2}\left(a_{n}^{0}-a_{n}\right)^{2}
\end{aligned}
$$

where $k=k_{B} \times T$ and $k_{B}$ is Boltzmann's Constant, $T$ is the temperature, and $L_{p}$ is the persistence length. Also based on the equi-partition theorem $\langle U\rangle=1 / 2 k_{B} T$, we can calculate the persistence length based on:

$$
L_{p}=\frac{L^{2}}{n^{2} \pi^{2} \operatorname{Var}\left(a_{n}\right)}
$$

where

$$
\operatorname{Var}\left(a_{n}\right)=\left\langle\left(a_{n}-a_{n}^{0}\right)^{2}\right\rangle
$$

The Fourier coefficients can be calculated as:

$$
a_{n} \cong \sqrt{\frac{2}{L}} \sum_{k=1}^{N} \theta_{k} S_{k} \cos \left(\frac{n \pi}{L} S_{k}^{\text {mid }}\right)
$$

where

$$
S_{k}^{m i d}=\Delta S_{1}+\Delta S_{2}+\cdots+\Delta S_{k-1}+\frac{1}{2} \Delta S_{k}
$$

As explained by Gittes, the measured length of a microtubule consists of an error due to the quality of our images, which is associated to the microscope resolution as well as the sample preparation. The uncertainly in measuring a length can also be associated to the possible drift of microtubules in the sample during the recording of the videos taken from our different samples. The uncertainly in microtubules' length can be obtained from the deviation from the real position along a microtubule. It can be expressed by a random distance $\varepsilon$, and the error in estimating the filament length can be calculated as:

$$
L^{\prime}=\sum_{k=1}^{N} \Delta s_{k} \cong \sum_{k=1}^{N} \Delta s_{0}\left[1+\frac{1}{2}\left(\frac{\varepsilon_{k+1}-\varepsilon_{k}}{\Delta s_{0}}\right)^{2}\right]
$$

And

$$
\frac{L^{\prime}}{L} \cong 1-\frac{\left\langle\varepsilon_{k}^{2}\right\rangle}{\Delta s_{0}^{2}}
$$

The technique to calculate $\left\langle\varepsilon_{k}\right\rangle$ is explicitly explained in Gittes [21]. In brief, it can be shown that

$$
\begin{aligned}
& \operatorname{Var}\left(a_{n}\right) \\
& =\left(\frac{L}{n \pi}\right)^{2} \frac{1}{L_{P}}+\frac{4}{L}\left\langle\varepsilon_{k}^{2}\right\rangle\left[1+(M-1) \sin ^{2}\left(\frac{n \pi}{2 M}\right)\right]
\end{aligned}
$$

and the $\left\langle\varepsilon_{k}^{2}\right\rangle$ can be calculated by the least-square curve fitting of $\operatorname{Var}\left(a_{n}\right)$ as a function of $n$. This error is different from one microtubule to another. In our study this error is measured and discussed in the following section.

\section{RESULTS}

To measure the persistence length of a single soybean microtubule in vitro, we incubated soybean tubulin with $10 \%$ Glycerol at $37^{\circ} \mathrm{C}$ and noticed that the time required for polymerization of soybean tubulin to reach to a typical length beyond $15 \mu \mathrm{m}$ is longer than the time for Bovine brain tubulin at the same experimental condition. Bovine brain tubulin with $10 \%$ Glycerol can be polymerized to long microtubules after incubation for $30 \mathrm{~min}$ utes. In contrast, we didn't observe any signs of polymerization initiation after 30 minutes of incubation in soybean samples. The focus of this study was not measuring the polymerization and dynamic parameters of a single soybean microtubule. However, we observed that soybean microtubules with longer length are reachable after incubation for two hours. Also, as compared with Bovine brain, the samples of soybean microtubules were much less populated, although tubulin was polymerized from the concentration of $5 \mathrm{mg} / \mathrm{ml}$. All this evidence supports the hypothesis that the nucleation and polymerization specifications of a soybean microtubule are different from those in Bovine brain. Therefore, as we observed, single soybean microtubules could freely fluctuate in response to the temperature in our samples without further dilution. 
So, unlike similar previous studies in measuring the flexural rigidity of microtubules, our microtubule samples were not stabilized, or the samples were not diluted with an antimitotic stabilizer agent such as paclitaxel. Figure 1 shows a single soybean microtubule viewed in one of our samples. The same soybean microtubule is viewed again in a different frame of the movie taken from the same sample. The two frames located side by side clearly show the bending of soybean microtubules. This is a small bending as in general microtubules are stiff.

To illustrate the microtubules' shape and monitor the deflection as a result of their response to the temperature, several points were selected along single microtubules, and the positions of digitalized points were monitored over time. To be consistent with the Gittes method in selecting the number of the digitalized points along a single microtubule, we assured that the length of each segment between two points did not exceed $4 \mu \mathrm{m}$. As explained by Gittes et al., for stiff bio-filaments such as microtubules that show small curvature as a result of thermal fluctuations, this segment length is appropriate to precisely reflect the bending. Therefore, as we set the limit for the average segment length to be almost $4 \mu \mathrm{m}$, we selected 7 points $(n=1, \cdots, 7)$ along an individual microtubule in our different samples. The coordinates associated to the positions of these points were recorded every 10 seconds. In this study we calculated the persistence length of seven individual soybean microtubules with different lengths.

The length of each microtubule and the error associated to each microtubule's length were calculated implementing Eqs.3 and 13. The Fourier coefficients, $a_{n}=$ $a_{1}, \cdots, a_{4}$, and as a last step the persistence length of the single microtubule, were obtained using Eqs.8-10. The results are summarized in Table 1.

In calculating the persistence length, the behavior of the variance of each mode was observed to be random, which indicates that our measurement was not associated with experimental noise. The typical behavior of the variance of modes that is expressed in Figure 2 shows an initial reduction in its value (solid dots) before rising. As the variance does not express a monotonic increase, the

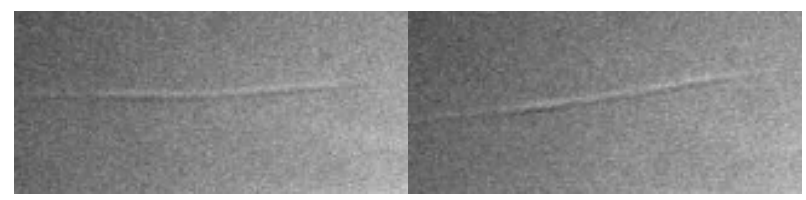

Figure 1. Represents two images of a single soybean microtubule in vitro taken by DIC microscopy at two different times. The above image consists of two selected images to demonstrate the shape of thermal fluctuations of a single microtubule in the field of view of the microscope.
Table 1. Persistence length of soybean microtubules with the different length between $15.71 \mu \mathrm{m}$ - $20.07 \mu \mathrm{m}$ polymerized from pure soybean tubulin in the presence of $10 \%$ Glycerol in $27^{\circ} \mathrm{C}$ measured from thermal fluctuations in shape method is estimated. In the second row, the value of the persistence length calculated from the first mode is expressed. Considering Eq.12, the relative difference between true length and the measured length can be calculated as $\frac{\left(L-L^{\prime}\right)}{L^{\prime}}=\frac{\left\langle\varepsilon_{k}^{2}\right\rangle}{\Delta \varepsilon_{0}^{2}}$. The $\left\langle\varepsilon_{k}^{2}\right\rangle$ can be calculated by the least-square curve fitting of $\operatorname{Var}\left(a_{n}\right)$ as a function of $\mathrm{n}$ as can be expressed in Figure 2. Also, as explained, the overall average segment length $\Delta s_{0}$ is $4 \mu \mathrm{m}$. The average of the length difference for our seven samples is $0.06 \%$. This percent difference is ignorable and has no significant effect on measuring the persistence length.

\begin{tabular}{cc}
\hline MT Length $(\mu \mathrm{m})$ & Persistence Length Mode 1 $(\mathrm{mm})$ \\
\hline 15.71 & 2.84 \\
17.19 & 6.19 \\
17.94 & 3.42 \\
19.34 & 4.23 \\
19.97 & 8.02 \\
20.07 & 4.38 \\
20.02 & 7.51 \\
\hline
\end{tabular}

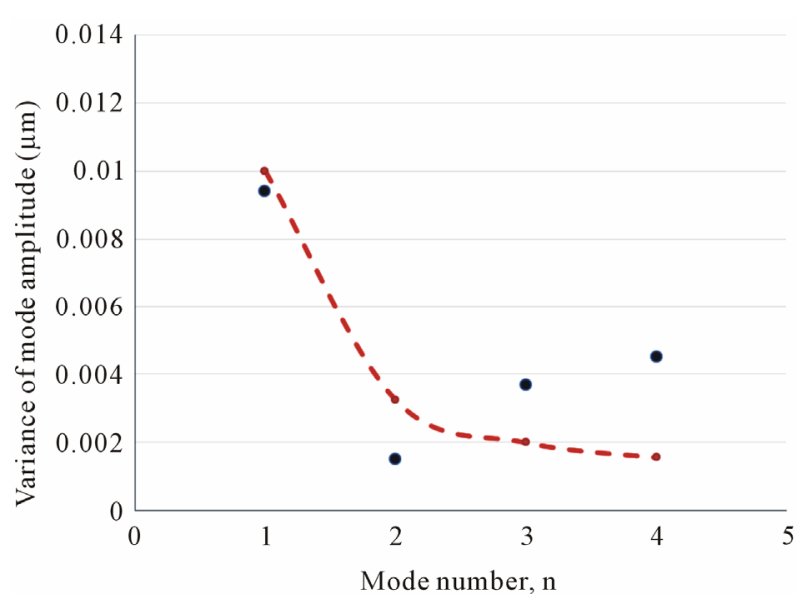

Figure 2. Solid dots: the variance of mode amplitude versus mode numbers. The dashed curve is the least square fit of Eq.14 essential for measuring errors in microtubules' length. The random behavior of the variance indicates that the bending is due to the microtubules' thermal fluctuations.

bending is related to the thermal fluctuations and not experimental noise.

Also, as expressed in Table 1, we measured the persistence length of seven soybean microtubules with the length between of $15 \mu \mathrm{m}-21 \mu \mathrm{m}$ and obtained a persistence length between $2.8 \mathrm{~mm}-8 \mathrm{~mm}$. The length dependency of persistence length and the liner fit is shown in Figure 3. 


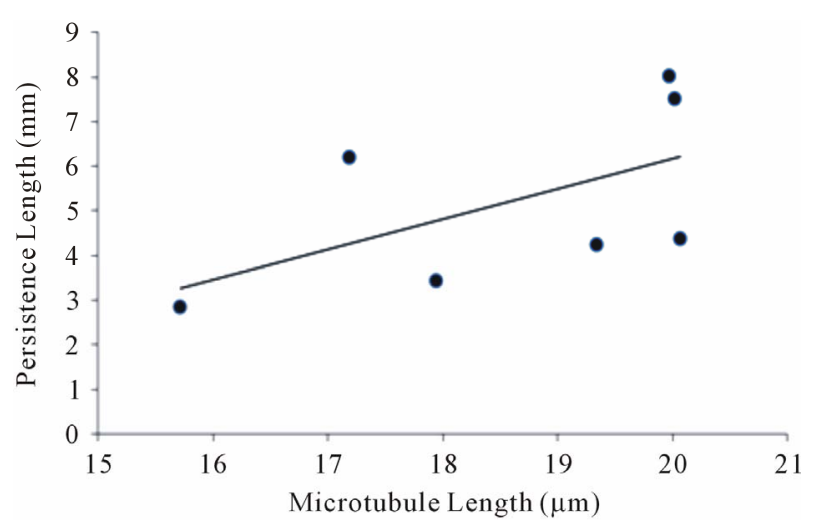

Figure 3. The solid dots represent the persistence length of 7 soybean microtubules plotted as a function of the length of each microtubule and the solid line is the linear fit.

\section{DISCUSSION}

While microtubules have been subject of many studies, the focus of many of the biomechanical researches has been on mammalian and neural microtubules rather than plant microtubules. To date, we know of many similarities among plant and mammalian microtubules such as stochastic behavior that can be observed in the dynamic of both types of microtubules [7,32]. However, plant microtubules are distinguished from mammalian microtubules in different areas, such as their unique pharmacological properties, different dynamic parameters, or different interaction with MAP [33]. Many of these contrasts are associated to significant differences and diversity of tubulin isotypes in the structure of plant microtubules as compared to mammalian microtubules.

The functional diversity of plant microtubules still needs to be further investigated from other perspectives. We chose to study one of the important mechanical properties of plant microtubules by examining the rigidity of a single soybean microtubule in vitro. Soybean microtubules are significant because they have a unique compositional structure in terms of $\beta$ tubulin isotypes. Although the $\beta$ tubulin isotype structure of soybean tubulin has not been accurately reported, the existence of three different beta isotypes ( $\beta$-I, $\beta$-II and pGE23) is confirmed [34]. In comparison with a mammalian neural tubulin such as Bovine Brain with $3 \% \beta$-I, $58 \% \beta$-II, $25 \% \beta$-III, and $13 \% \beta$-IV, or a non-neural mammalian tubulin such as MCF7 cell tubulin with $22 \% \beta$-I, $0 \% \beta$-II, $51 \% \beta$-III, and $24 \% \beta$-IV, soybean tubulin has a different structural composition. As we run a parallel experiment with Bovine Brain tubulin, in the first step we noticed polymerization differences between these two types of tubulin (unpublished results).

We measured the persistence length of 7 single soybean microtubules with different lengths in vitro, through a thermally driven fluctuation method in curvature. As reflected in Table 1, the experimental error associated with the length of each single soybean microtubule is small. Therefore, the variation in calculated persistence length is not significant. The estimated persistence length for soybean microtubules shows a length dependency as expressed in Figure 3. The average persistence length obtained from the first mode is $5.22 \mathrm{~mm}$. This value is higher than the average persistence length we measured in our previous study for the MCF7 and bovine brain microtubules under the same experimental condition [31]. In contrast with our previous study, the soybean microtubules' length changes broadly between $15-10 \mu \mathrm{m}$ in our different exponential samples. Considering the length dependency of the persistence length of microtubules, the higher value of the average persistence length can be linked to the fact that length of microtubules changes up to $4.5 \mu \mathrm{m}$ from one another. The different value of the persistence length in soybean microtubules can be associated to the different beta isotype structure of soybean microtubules. So far, many differences in functionality of plant microtubules, ranging from their dynamic characters to their mechanism of interacting with anti-mitotic drugs, are linked to the existence of different beta tubulin isotypes in their structure. It is reasonable that differences in the rigidity of soybean microtubules can also be caused by these compositional differences. In addition, the origin of the difference between persistence length in Bovine brain, MCF7, and soybean microtubules can be associated with the different number of proto-filaments in the structure of a single soybean microtubule as compared to mammalian microtubules. While the architecture of soybean microtubules in terms of the number of proto-filament still needs to be investigated, the higher value of the persistence length can be the result of the higher number of protofilaments in the structure of microtubules. Another possible source of differences in rigidity can be correlated to the existence of the impurity in soybean tubulin used in this experiment. The Kit of soybean tubulin consisted of tubulin with $90 \%$ purification. The existence of impurity, including MAPs, can cause a difference in persistence length as compared with those in the Bovine brain percentage of purification.

This study measures the persistence length of a single soybean microtubule as one of the plant species. This measurement can support the distinguished role of $\beta$ tubulin isotypes' compositional diversity in the functional diversity of microtubules, which is not limited to the change in dynamic characters, but includes the mechanical properties as well.

One of the features distinguishing plants is the cell walls. Unlike animals in which actin filaments are involved in cell shape and plasma membrane, microtubules are dominant elements in plasma membrane in plant cells $[35,36]$. The persistence length of a single soybean's 
microtubules measured in this study and the length dependency observed in this study can be significant for development of the plant cell walls and therefore plant cell shape.

Our results suggest that the diversity in the compositional structure of microtubules can not only regulate the dynamic of microtubules, but affect the mechanical properties as well, and support the fundamental differences between plant and mammalian microtubules which are required for their different functionality. While this study was conducted in vitro, for a better understanding of mechanical properties of soybean microtubules, further experiments are required to measure this rigidity in vivo. The results can be further confirmed by implementing different methods such as measuring the thermal fluctuation of grafted microtubules or optical trapping techniques. Applying different methods of measuring the persistence length together with examining the structure of soybean microtubules by the method of electron microscopy can reveal the possible source of differences among soybean and other microtubules [37].

\section{ACKNOWLEDGEMENTS}

This research was funded by the Physics Department of Seton Hall University.

\section{REFERENCES}

[1] Hyams, J.S and Lloyd, C.S. (1994) Microtubules. WileyLiss, Inc., New York.

[2] Howard, J. (2001) Mechanics of motor proteins and the cytoskeleton. Sinauer Associates, Sunderland.

[3] Hill, T.L. (1981) Microfilament or microtubule assembly or disassembly against a force. Proceedings of the $\mathrm{Na}$ tional Academy of Sciences of the United States of America, 78, 5613-5617. http://dx.doi.org/10.1073/pnas.78.9.5613

[4] Dixit, R. and Cyr, R.J. (2004) The cortical microtubule array: From dynamics to organization. Plant Cell, 16, 2546-2552. http://dx.doi.org/10.1105/tpc.104.161030

[5] Cyr, R.J. (1994) Microtubules in plant morphogenesis: Role of the cortical array. Annual Review of Cell and Developmental Biology, 10, 153-180.

http://dx.doi.org/10.1146/annurev.cb.10.110194.001101

[6] Cyr, R.J. and Palevitz, B.A. (1995) Organization of cortical microtubules in plant cells. Current Opinion in Cell Biology, 7, 65-71. http://dx.doi.org/10.1016/0955-0674(95)80046-8

[7] Moore, R.C., Zhang, M., Cassimeris, L. and Cyr, R.J. (1997) In vitro assembled plant microtubules exhibit a high state of dynamic instability. Cell Motility and the Cytoskeleton, 38, 278-286.

http://dx.doi.org/10.1002/(SICI)1097-0169(1997)38:3<27 8::AID-CM6>3.0.CO;2-1

[8] Wasteneys, G.O. (2002) Microtubule organization in the green kingdom: Chaos or self-order? Journal of Cell Science, 115, 1345-1354.

[9] Kirschner, M.W. and Mitchison, T. (1986) Microtubule dynamics. Nature, 324, 621.

http://dx.doi.org/10.1038/324621a0

[10] Walker, R.A., O’Brien, E.T. and Pryer, N.K. (1988) Dynamic instability of individual, MAP-free microtubules analyzed by video light microscopy: Rate constants and transition frequencies. The Journal of Cell Biology, 107, 1437-1448. http://dx.doi.org/10.1083/jcb.107.4.1437

[11] Shibaoka, H. (1994) Plant hormone-induced changes in the orientation of cortical microtubules: Alterations in the cross-linking between microtubules and the plasma membrane. Annual Review of Plant Physiology and Plant Molecular Biology, 45, 527-544.

http://dx.doi.org/10.1146/annurev.pp.45.060194.002523

[12] Komarokova, Y.A., Vorobjev, I.A. and Borisy, G.G. (2002) Life cycle of MTs: persistent growth in the cell interior, asymmetric transition frequencies and effects of the cell boundary. Journal of Cell Science, 115, 35273529.

[13] Govindan, B. and Spillman Jr., W.B. (2004) Steady states of a microtubule assembly in a confined geometry. Physical Review E, 70, 32901-32904.

http://dx.doi.org/10.1103/PhysRevE.70.032901

[14] Panda, D., Miller, H.P., Banerjee, A., Luduena, R.F., et al. (1994) Microtubule dynamics in vitro are regulated by the tubulin isotype composition. Proceedings of the $\mathrm{Na}$ tional Academy of Sciences, 91, 11358-11362. http://dx.doi.org/10.1073/pnas.91.24.11358

[15] Newton, C.N., DeLuca, J.G., Himes, R.H., Miller, H.P., et al. (2002) Intrinsically slow dynamic instability of HeLa cell microtubules in vitro. The Journal of Biological Chemistry, 277, 42456-42462. http://dx.doi.org/10.1074/jbc.M207134200

[16] Morejohn, L.C. (1991) The molecular pharmacology of plant tubulin and microtubules. In: Lloyd, C.W., Ed., The Cytoskeletal Basis of Plant Growth and Form, Academic Press, Inc., San Diego, 29-44.

[17] Banerjee, A. (2002) Increased levels of tyrosinated a-, $\beta$ III-, and $\beta$ IV-tubulin isotypes in paclitaxel-resistant MCF-7 breast cancer cells. Biochem. Biochemical and Biophysical Research Communications, 293, 598-601. http://dx.doi.org/10.1016/S0006-291X(02)00269-3

[18] Shalli, K., Brown, I., Heys, S.D. and Schofield, A.C. (2005) Alterations of $\beta$-tubulin isotypes in breast cancer cells resistant to docetaxel. The FASEB Journal, 19, 1299-1301.

[19] Huzil, J.T., Chen, K., Kurgan, L. and Tuszynski, J.A. (2007) The roles of $\beta$-tubulin mutations and isotype expression in acquired drug resistance. Cancer Information, 3, 159-181

[20] Chen, K., Huzil, J.T., Freedman, H., et al. (2008) Identification of tubulin drug binding sites and prediction of relative differences in binding affinities to tubulin isotypes using digital signal processing. Journal of Molecular Graphics and Modelling, 27, 497-505. http://dx.doi.org/10.1016/j.jmgm.2008.09.001 
[21] Gittes, F., Mickey, B., Nettleton, J. and Howard, J. (1993) Flexural rigidity of microtubules and actin filaments measured from thermal fluctuations in shape. The Journal of Cell Biology, 120, 923-934. http://dx.doi.org/10.1083/jcb.120.4.923

[22] Venier, P., Maggs, A.C., Carlier, M.F., et al. (1994) Analysis of microtubule rigidity using hydrodynamic flow and thermal fluctuations. The Journal of Biological Chemistry, 269, 13353-13360.

[23] Kurachi, M., Hoshi, M. and Tashiro, H. (1995) Buckling of a single microtubule by optical trapping forces: Direct measurement of microtubule rigidity. Cell Motility and the Cytoskeleton, 30, 221-228. http://dx.doi.org/10.1002/cm.970300306

[24] Kawaguchi, K., Ishiwata, S. and Yamashita, T. (2008) Temperature dependence of the flexural rigidity of single microtubules. Biochemical and Biophysical Research Communications, 366, 637-642. http://dx.doi.org/10.1016/j.bbrc.2007.11.162

[25] Kikumoto, M., Kurachi, M., Tosa, V., et al. (2006) Flexural rigidity of individual microtubules by buckling force with optical traps. Biophysical Journal, 90, 1687-1696. http://dx.doi.org/10.1529/biophysj.104.055483

[26] Felgner, H., Frank, R. and Schliwa, M. (1996) Flexural rigidity of microtubules measured with the use of optical tweezers. Journal of Cell Science, 109, 509-516.

[27] Mickey, B. and Howard, J. (1995) Rigidity of microtubules is increased by stabilizing agents. The Journal of Cell Biology, 130, 909-917. http://dx.doi.org/10.1083/jcb.130.4.909

[28] Hawkins, T., Mirigian, M., Selcuk, Y.M. and Ross, J.L. (2010) Mechanics of microtubules, Journal of Biomechanics, 43, 23-30. http://dx.doi.org/10.1016/j.jbiomech.2009.09.005

[29] Pampaloni, F., Lattanzi, G., Jonás, A., Surrey, T., Frey, E. and Florin, E.L. (2006) Thermal fluctuations of grafted microtubules provide evidence of a length-dependent persistence length. Proceedings of the National Academy of Sciences of the United States of America, 103, 1024810253. http://dx.doi.org/10.1073/pnas.0603931103

[30] Kawaguchi, K. and Yamaguchi, A. (2010) Temperature dependence rigidity of non-taxol stabilized single microtubules. Biochemical and Biophysical Research Communications, 402, 66-69. http://dx.doi.org/10.1016/j.bbrc.2010.09.112

[31] Feizabadi, M.S., Mutafopulos, K. and Behr, A. (2011) Measuring the persistence length of MCF7 cell microtubules in vitro. Journal of Biotechnology, 6, 882-887. http://dx.doi.org/10.1002/biot.201000465

[32] Vassileva, V.N., Fujii, Y. and Ridge, R.W. (2005) Microtubule dynamics in plants. Plant Biotechnology, 22, 171178. http://dx.doi.org/10.5511/plantbiotechnology.22.171

[33] Goddard, R.H., Wick, S.M. and Silflow, C.D., et al. (1994) Microtubule components of the plant cell cytoskeleton. Plant Physiology, 104, 1-6.

[34] Luduena, R. (1993) Are tubulin isotypes functionally significant. Molecular Biology of the Cell, 4, 445-457. http://dx.doi.org/10.1091/mbc.4.5.445

[35] Wasteneys, G.O. (2004) Progress in understanding the role of microtubules in plant cells. Current Opinion in Plant Biology, 7, 651-660. http://dx.doi.org/10.1016/j.pbi.2004.09.008

[36] Wasteneys, G.O. and Fujita, M. (2006) Establishing and maintaining axial growth: Wall mechanical properties and the cytoskeleton. Journal of Plant Research, 119, 510. http://dx.doi.org/10.1007/s10265-005-0233-3

[37] Simon, J.R. and Salmon, E.D. (1990) The structure of microtubule ends during the elongation and shortening phases of dynamic instability examined by negative-stain electron microscopy. Journal of Cell Science, 96, 571582. 